\title{
Does MRS Lactate Peak Correlate with Lactate in the CSF and Blood?
}

\author{
Simone Shibao ${ }^{1}$ Maria C. G. Otaduy ${ }^{1}$ Fernando Kok ${ }^{2}$ Claudia C. Leite ${ }^{1,3}$ \\ ${ }^{1}$ Department of Radiology, School of Medicine, Universidade de São \\ Paulo, São Paulo, Brazil \\ 2 Department of Neurology, School of Medicine, Universidade de São \\ Paulo, São Paulo, Brazil \\ ${ }^{3}$ Department of Radiology, School of Medicine, University of North \\ Carolina, Chapel Hill, North Carolina, United States \\ Address for correspondence Claudia C. Leite, MD, PhD, Av. Dr. Ovídio \\ Pires Campos S/N, Inrad portaria 5-Ressonancia Magnética, São Paulo, \\ SP, Brazil (e-mail: Claudia.leite@hc.fm.usp.br). \\ J Pediatr Neuroradiol 2015;4:1-6.
}

\begin{abstract}
Purpose Cerebrospinal fluid (CSF) or brain parenchyma lactate detection is important for the diagnosis of some diseases with aerobic cellular metabolism compromise. Our purpose is to correlate intraventricular magnetic resonance spectroscopy (MRS) lactate detection and quantification to CSF and blood lactate concentration.

Methods Twenty-one patients ( 13 females; mean age 5 years) suspected of having mitochondrial disorders underwent proton MRS with point-resolved spectroscopy $(\mathrm{TE}=144 \mathrm{~ms})$. The volume of interest was positioned in the lateral ventricles, and LCModel was used for the MRS lactate peak detection and quantification. CSF and venous blood samples were obtained for lactate quantification immediately after MRS. Comparisons between MRS, CSF, and blood lactate detection and quantification were performed. $p<0.05$ was considered significant.

Results In our series, CSF lactate levels were high in 11 patients (52\%) and blood serum lactate levels were high in 3 patients (14\%). MRS was able to detect a lactate peak in all

\section{Keywords}

- magnetic resonance spectroscopy

- lactate peak

- CSF lactate concentration

- blood lactate concentration

- mitochondrial disorders patients. A positive correlation between MRS lactate quantification and CSF lactate was observed (Pearson correlation coefficient $=0.750 ; p<0.0001$ ). Blood lactate did not correlate with lactate levels in the lateral ventricle measured by MRS or direct determinations of CSF lactate concentration. When noting the lactate quantification obtained by MRS, only 8 patients (as opposed to 14) presented higher values than the established normality threshold of $1.7 \mathrm{mmol} / \mathrm{L}$. Considering CSF puncture concentration as the gold standard, we obtained $64 \%$ of sensitivity and $90 \%$ of specificity for MRS quantification.

Conclusion If MRS shows increased lactate levels in the ventricles, CSF puncture is not needed for lactate increase confirmation.
\end{abstract}

\section{Introduction}

Lactate cerebrospinal fluid (CSF) biochemical analysis is an invasive procedure routinely required during evaluation and monitoring of many neurological diseases. CSF lactate is considered an indicator of central nervous system cellular oxidative metabolism and many attempts have been made to set a reference limit of its concentration in children. ${ }^{1,2}$ CSF concentration may change within a short period of time, while blood lactate concentrations may not yet be affected. There is a lack of good correlation between direct measurement of CSF and plasma lactate that is attributable to the received

June 23, 2014

accepted after revision

May 11, 2015

published online

September 30, 2015
Copyright $\odot 2015$ by Georg Thieme

Verlag KG, Stuttgart - New York
DOI http://dx.doi.org/ 10.1055/s-0035-1564663. ISSN 1309-6680. 
blood-brain barrier, which prevents a fast equilibrium between those two fluid compartments. ${ }^{3}$ Additionally, venous plasma determination can be affected by the sampling conditions, such as patient agitation, screaming, and prolonged tourniquet time.

Magnetic resonance spectroscopy (MRS) allows a noninvasive detection of lactate peak (LP) in the brain parenchyma and in the ventricular system, while postprocessing of MRS data using the LCModel software enables its quantification. ${ }^{4}$ Previous studies have tried to show a correlation between increase in the LP detected on MRS and the lactate levels in CSF and/or blood, but the methodologies were diverse and the results were sometimes inconsistent. Most of the studies evaluated only MRS with voxels located in the brain parenchyma. $^{\text {5-10 }}$

Mitochondrial disorders can be a good model for a respiratory cell compromise disorder. In mitochondrial disorders, patients with central nervous system involvement usually present with increased lactate in the CSF and sometimes in the plasma, with lactate detection being an important tool in the work-up of these conditions. ${ }^{11,12}$

The purpose of this study, which was performed in patients with suspected mitochondrial disorders, was to complete a systematic comparison of intraventricular MRS lactate detection and quantification and the determination of CSF and venous lactate performed immediately after MRS.

\section{Methods}

\section{Subjects}

Twenty-one patients with clinical suspicion of mitochondrial disorders (13 female and 8 male; mean age: 5 years; range from 9 months to 18 years of age) were included in this prospective study in a 1-year period from November 2005 to November 2006. Our Institutional Review Board approved this protocol, and all patients or their legal representative gave written informed consent to participate in the study.

\section{Magnetic Resonance Imaging and Proton Magnetic Resonance Spectroscopy}

All subjects were scanned on a whole-body unit operating at 1.5 T (Signa Horizon LX; GE Medical Systems, Milwaukee, Wisconsin, United States) with a standard quadrature head coil. Magnetic resonance imaging (MRI) and MRS were performed at the same time in the initial clinical investigation for mitochondrial disorders. To locate the MRS volume of interest (VOI), an axial fast spin-echo T2-weighted image was performed, with the following parameters: repetition time/echo time (TR/TE): 3,000/88.8 ms, echo train length: 22 , number of excitations $(\mathrm{NEX})=1$, slice thickness $10 \mathrm{~mm}$. Single-voxel MRS was obtained by point-resolved spectroscopy (TR/TE: 1,500/144 ms, 8 phase-cycle steps, and 96 to 192 acquisitions, depending on voxel size). The MRS VOI was placed in the lateral ventricles (-Fig. 1A).

The VOI was individually adjusted by a 12-year experienced neuroradiologist to minimize gray/white matter partial volume effects. Its size was variable depending on the size of the lateral ventricles and ranged from 6.4 to $26.9 \mathrm{~cm}^{3}$ (mean voxel size: $11.7 \mathrm{~cm}^{3}$ ). Before spectra were acquired, a VOI shimming procedure was performed to ensure adequate field homogeneity, which typically resulted in a water resonance line no wider than $6 \mathrm{~Hz}$ (full width at half maximum). This step was followed by water suppression adjustment with selective inversion of water resonance.

\section{Metabolite Postprocessing and Quantification}

Raw MRS data of the patients were processed offline on a personal computer with LCModel software (Version 6.1.), an automated and noninteractive quantification tool for MRS data. The in vivo spectrum was analyzed in the frequency domain as linear combination of a complete

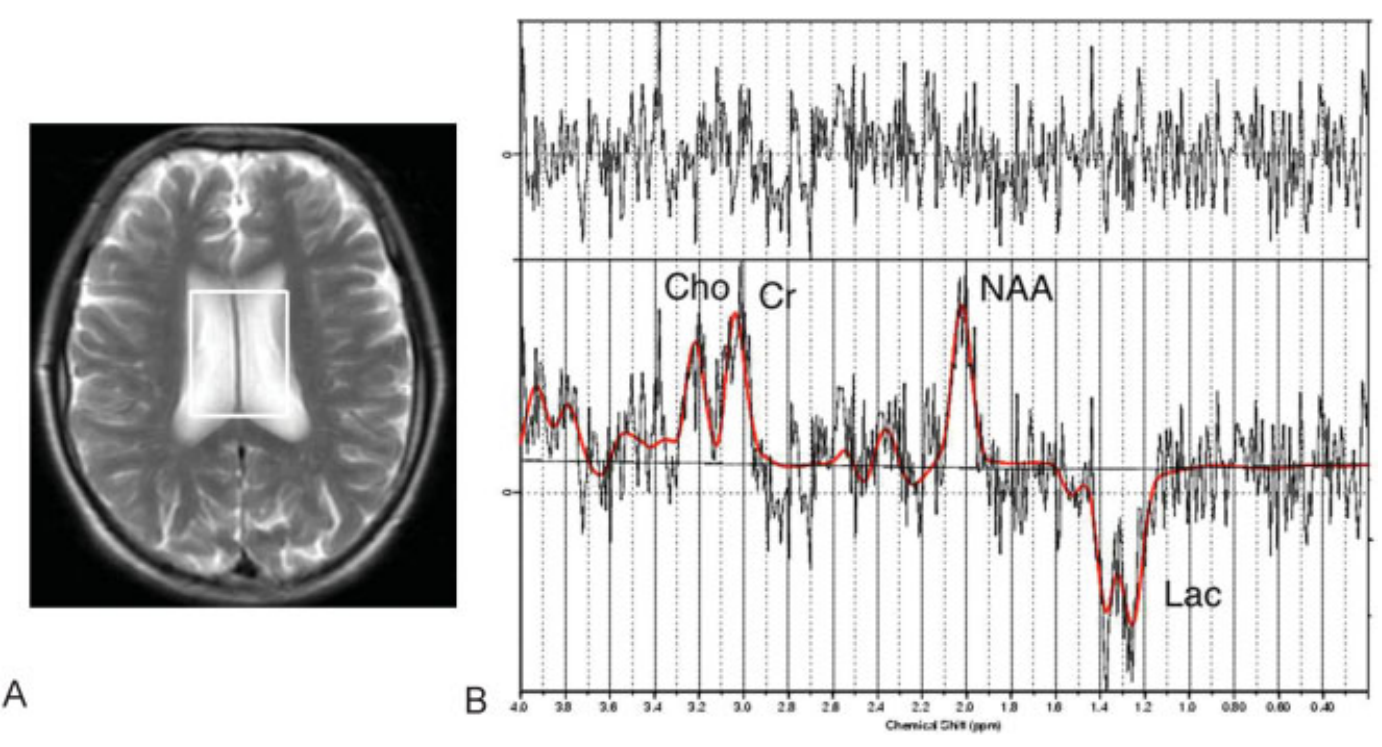

Fig. 1 MRI and MRS in (A) axial T2-weighted MRI show typical VOI for MRS location in the lateral ventricles. MRS (B) showing the inverted lactate peak and LCModel fit (red curve) to the original signal (black). Above the spectrum the residual after fitting. 
spectral database of metabolic in vitro solutions. The LP was identified as an inverted doublet peak (coupling constant $\mathrm{J}=7 \mathrm{~Hz}$ ) at $1.33 \mathrm{ppm}$ (-Fig. 1B). Absolute metabolic concentration was estimated using the water signal in the lateral ventricles as an internal reference. We corrected for water T1 and T2 relaxation assuming a T1 of 2.9 second and a T2 of 1.5 second. ${ }^{13,14}$ The VOI was placed in the lateral ventricle avoiding contamination with brain tissue; therefore, for conversion to concentration units we assumed the water concentration in $\operatorname{CSF}(53,889 \mathrm{mmol} / \mathrm{L})$ that had been previously reported in the literature. ${ }^{15}$ To our knowledge, there is only one report of in vivo lactate CSF relaxation times (measured intraventricular), which presented large differences in CSF lactate T2, ranging from $323 \mathrm{~ms}$ to 1,122 $\mathrm{ms}$, depending on patient/pathology, ${ }^{16}$ and there is no report of CSF lactate T1. Given this uncertainty, we opted not to correct for lactate relaxation times. However, LCModel quantification relies on comparison with an in vitro metabolite basis set measured with the same TE and subject to the same transverse relaxation effects; therefore, the obtained concentration values already take into account the T2 effects, assuming that the lactate T2 in ventricular CSF is similar to the lactate T2 in the in vitro solution of the LCModel basis dataset.

\section{Biochemical Analysis}

Immediately after MRI and MRS conclusion, CSF and blood samples were obtained for determination of lactate levels. CSF was obtained by lumbar puncture in strict aseptic conditions and it was immediately cooled and sent to the laboratory, where it was stored in a thermal recipient. Blood was collected in a fluoride tube from a free-flowing peripheral vein and centrifuged. Lactate determination in both CSF and plasma was performed immediately by a colorimetric enzymatic standard assay. The upper reference level that was used for lactate CSF was $1.7 \mathrm{mmol} / \mathrm{L}$ (15.3 $\mathrm{mg} / \mathrm{dL}$ ) (2) and that for plasma was $2.1 \mathrm{mmol} / \mathrm{L}(19 \mathrm{mg} / \mathrm{dL})$.

\section{Statistical Analysis}

We analyzed the correlation between lactate quantified by MRS, CSF, and blood sampling. Statistical analysis of the data was performed using the Pearson correlation test. A $p$-value of $<0.05$ was considered statistically significant.

\section{Results}

- Table 1 presents the results of lactate detection and quantification by MRS, CSF, and venous concentration. In

Table 1 Detection of lactate by MRS, blood, and cerebrospinal fluid

\begin{tabular}{|l|l|l|l|l|l|}
\hline Patient & $\begin{array}{l}\text { MRS lactate } \\
\text { (mmol/L) (CRLB in \%) }\end{array}$ & $\begin{array}{l}\text { CSF lactate } \\
\text { (mmol/L) }\end{array}$ & $\begin{array}{l}\text { CSF lactate } \\
\text { (mg/dL) }\end{array}$ & $\begin{array}{l}\text { Plasma lactate } \\
\text { (mmol/L) }\end{array}$ & $\begin{array}{l}\text { Plasma lactate } \\
\text { (mg/dL) }\end{array}$ \\
\hline 1 & $1.26(41)$ & 2.45 & 22.1 & 1.01 & 9.1 \\
\hline 2 & $1.47(17)$ & 1.37 & 12.3 & 0.89 & 8.0 \\
\hline 3 & $0.74(47)$ & 1.24 & 11.2 & 0.71 & 6.4 \\
\hline 4 & $0.26(124)$ & 1.44 & 13.0 & 1.21 & 10.9 \\
\hline 5 & $5.02(13)$ & 3.87 & 34.9 & 1.64 & 14.8 \\
\hline 6 & $1.39(32)$ & 1.01 & 9.1 & 0.73 & 6.6 \\
\hline 7 & $0.95(22)$ & 1.55 & 14.0 & 0.89 & 8.0 \\
\hline 8 & $3.13(24)$ & 3.82 & 35.4 & 5.88 & 53.0 \\
\hline 9 & $2.37(33)$ & 1.74 & 15.7 & 0.89 & 8.0 \\
\hline 10 & $2.54(20)$ & 3.82 & 34.4 & 1.39 & 12.5 \\
\hline 11 & $1.87(32)$ & 1.68 & 15.1 & 1.44 & 13.0 \\
\hline 12 & $6.35(13)$ & 3.36 & 30.3 & 1.39 & 12.5 \\
\hline 13 & $1.56(23)$ & 0.94 & 8.5 & 3.79 & 34.1 \\
\hline 14 & $1.56(25)$ & 1.69 & 15.2 & 1.43 & 12.9 \\
\hline 15 & $0.42(11)$ & 1.48 & 13.3 & 1.68 & 15.1 \\
\hline 16 & $1.19(20)$ & 1.81 & 16.3 & 1.00 & 9.0 \\
\hline 17 & $3.68(16)$ & 3.91 & 35.2 & 2.25 & 20.3 \\
\hline 18 & $1.09(39)$ & 1.92 & 17.3 & 1.25 & 11.3 \\
\hline 19 & $2.65(13)$ & 3.77 & 34.0 & 1.33 & 12.0 \\
\hline 20 & $1.30(23)$ & 1.83 & 16.5 & 1.60 & 14.4 \\
\hline 21 & $1.16(21)$ & 1.70 & 15.3 & 2.10 & 18.9 \\
\hline
\end{tabular}

Abbreviations: CRLB, Cramér-Rao lower bound; CSF, cerebrospinal fluid; MRS, proton spectroscopy.

Note: Lactate CSF and plasma concentrations marked in bold are above the respective thresholds considered normal for these sampling techniques. 
11 patients (52\%), lactate was elevated in CSF and in 3 (14\%) individuals in plasma. Concomitant lactate increase in the CSF and blood was only noted in two patients (10\%). LCModel was able to detect a nonzero LP for all patients. As evidenced in - Table 1, some patients (patients 3, 4, and 15) with lower CSF lactate concentration presented a very high error (Cramér-Rao lower bound [CRLB] > 45\%) associated with lactate quantification. Lactate concentrations quantified by MRS varied from 0.26 to $6.35 \mathrm{mmol} / \mathrm{L}$ (median $1.47 \pm 1.51 \mathrm{mmol} / \mathrm{L}$ ). The corresponding CRLB varied from 13 to $124 \%$ (median $23 \pm 29 \%$ ).

Considering CSF lactate concentration obtained from puncture as the gold standard, our MRS results of the ventricles presented a sensitivity of $64 \%$ and a specificity of $90 \%$, using the same threshold as in CSF concentration (1.7 $\mathrm{mmol} / \mathrm{L}$ ). If we would use a threshold of $1.0 \mathrm{mmol} / \mathrm{L}$, the sensitivity would be $100 \%$ and the specificity would decrease to $40 \%$.

The dispersion graph between MRS LP quantification in the lateral ventricles and CSF lactate concentration is shown in - Fig. 2. Statistical analysis of the data using the Pearson correlation demonstrated a significant correlation between MRS LP quantification in the lateral ventricles and CSF lactate values (Pearson correlation coefficient $=0.750$; $p<0.0001)$. -Fig. 2 also demonstrates the dispersion graph of MRS LP in the lateral ventricles and blood lactate concentration. No correlation was obtained for MRS LP and blood lactate concentration (Pearson correlation coefficient $=0.221 ; p=0.338$ ).

The CSF lactate level was normal in one out of the three patients who presented increased lactate blood plasma levels. The dispersion graph between CSF and blood lactate concentrations is shown in - Fig. 3. No correlation was observed for CSF and blood lactate (Pearson correlation coefficient $=0.334 ; p=0.139$ ).

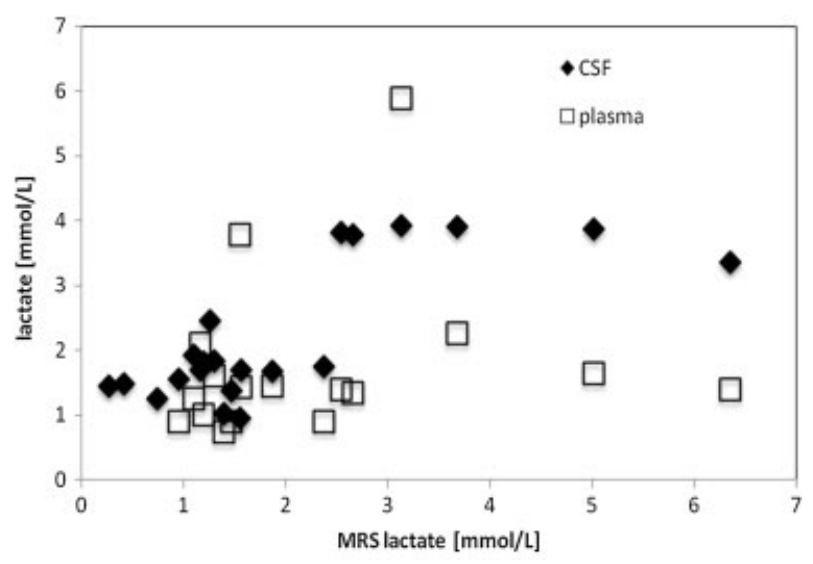

Fig. 2 Dispersion graph between MRS lactate quantification and lactate concentrations in mmol/L found in CSF (black diamonds) and in plasma (white squares). Statistical analysis of the data using Pearson correlation of data obtained by MRS and CSF lactate demonstrated a significant correlation with CSF lactate $(R=0.75 ; p<0.0001)$ but not with the plasma lactate $(R=0.221 ; p=0.338)$.

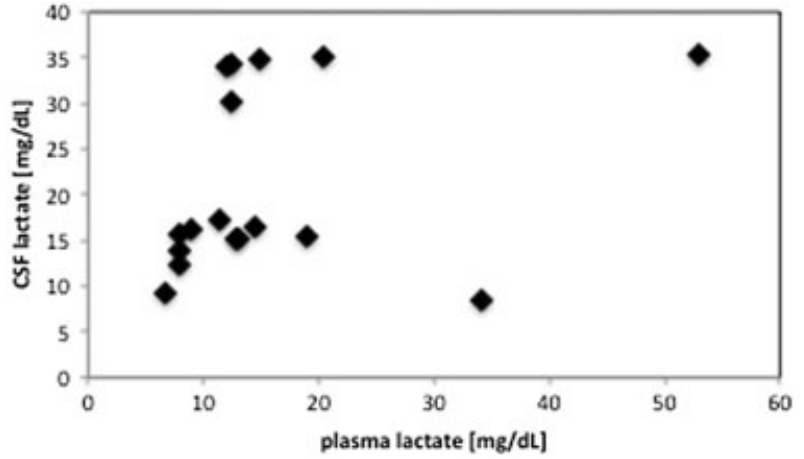

Fig. 3 Dispersion graph between CSF and serum lactate concentration $(\mathrm{mg} / \mathrm{dL})$. No correlation was obtained for these two variables. Pearson correlation coefficient $=0.334 ; p=0.139$.

\section{Discussion}

Lactate detection is a marker of impaired aerobic cellular metabolism and is commonly seen in mitochondrial disorders. The presence of lactate can be evaluated by blood analysis, CSF sampling, and MRS. Previous reports have shown that MRS is a useful tool for the noninvasive detection of an LP in the brain parenchyma and in the ventricles. $6,8,12$ Isobe et $\mathrm{al}^{17}$ performed an in vitro study that showed a correlation between lactate concentration in a phantom and its quantification by MRS, and they demonstrated the reliability of MRS in LP detection. In our study of patients suspected of having mitochondrial disorders, we were able to quantify an LP by MRS in the lateral ventricles of all studied patients. Considering a normality upper limit of $1.7 \mathrm{mmol}$ for MRS (the same used for CSF threshold), lactate was increased only in 8 patients, while it was elevated in the CSF of 11 patients and in the blood of 3 patients. Therefore, our data demonstrate that even patients presenting with normal CSF or blood lactate levels can have a detectable and quantifiable MRS LP.

A discrepant correlation between the results of MRS lactate detection and CSF lactate levels has been described in the literature. ${ }^{6-8,10}$ The methodology in the previous papers varied with VOI in different locations (parenchyma vs. ventricle) and different MRS techniques. Furthermore, the time interval between the MRS and blood or CSF samples was not mentioned or was not homogeneous, and the disease phase varied, which may in part explain the differences between MRS, blood, and CSF lactate detection. Chi et $\mathrm{al}^{6}$ studied 14 mitochondrial disease patients that presented LP in the brain parenchyma MRS; of those, 5 had also undergone a CSF sampling, and the CSF was positive for lactate in only 3 patients. The concomitant MRS LP elevation in the lateral ventricles and CSF lactate concentration increase was described in a small series $(n=3)$ by Feng et al. ${ }^{8}$ Boddaert et al ${ }^{7}$ used MRS and detected an LP in the brain, though the CSF lactate level was negative in 11 patients and the blood level was positive in 2 out of 9 patients that had blood sampling. Our results are in contrast to the data from Cross et al, ${ }^{10}$ who were able to detect an MRS LP in the brain parenchyma only when the CSF lactate level was above $4.0 \mathrm{mmol} / \mathrm{L}$. In our study, 
we could observe MRS LP even when the CSF lactate concentration was as low as $0.94 \mathrm{mmol} / \mathrm{L}(8.5 \mathrm{mg} / \mathrm{dL})$. However, when considering in MRS the same threshold as used for CSF lumbar puncture $(>1.7 \mathrm{mmol} / \mathrm{L})$, MRS detection presented only a sensitivity of $70 \%$. This lack of sensitivity might be related to a matter of low concentration, since the median lactate CSF concentration of the patients in which MRS failed to detect an elevated LP (above $1.7 \mathrm{mmol} / \mathrm{L}$ ) was $2.0 \pm 0.3$ $\mathrm{mmol} / \mathrm{L}$, while patients with an elevated MRS LP presented a higher concentration $(3.5 \pm 0.8 \mathrm{mmol} / \mathrm{L})$. This indicates that lactate concentration is after all a limiting factor for accurate MRS quantification.

In our sample, the blood lactate level was increased in three patients, but the LP detected on MRS was elevated in only two of these patients. In the other patient, lactate detected by MRS was $1.6 \mathrm{mmol} / \mathrm{L}$, just below the threshold of normality. Chi et $\mathrm{al}^{6}$ reported on a series of patients with mitochondrial disorders and found an LP on MRS in the brain parenchyma of 14 cases, though only 12 of the patients presented with an increased blood lactate level. Dinopoulos et $\mathrm{al}^{9}$ presented a series of 7 out of 17 patients with detected brain MRS LP and normal plasma lactate levels. Our patient series exclusively included patients who were suspected of having mitochondrial diseases during the initial work-up, as in the chronic phases of the disease the LP may not be detected. $8,11,18$

One point that should be discussed in the evaluation of LP by MRS is the VOI size. The smaller the VOI, the lower the signal-to-noise ratio of the spectrum and, therefore, the lower the chance of detecting a quantifiable lactate signal. The smallest voxel size for which an LP was observed in our series was $6.4 \mathrm{~mL}$ (patient 9). In this patient, the CSF concentration was on the borderline $(1.7 \mathrm{mmol} / \mathrm{L})$ and we obtained an MRS value of $2.37 \mathrm{mmol} / \mathrm{L}$, demonstrating that even with a small voxel size, the signal-to-noise ratio was not a preventing factor for lactate quantification.

The high specificity obtained for the MRS quantification is encouraging. It means that patients presenting an elevated LP already in MRS might not need to suffer from the more invasive procedure of CSF analysis by lumbar puncture.

A limitation of our lactate quantification by MRS is that we were assuming that the MRS voxel would contain only CSF without contamination from surrounding brain tissue. Even if care was taken to place and size the MRS voxel in the axial plane within the ventricle limits, we should remember that the rectangular voxel was $10 \mathrm{~mm}$ thick, thereby preventing a total exclusion of brain tissue. As a result, in most of our intraventricular spectra, we could also quantify other brain tissue metabolites, such as $\mathrm{N}$-acetylaspartate, creatine, and choline. Water content and water relaxation properties are different in brain tissue than in CSF; therefore, a partial volume effect with brain tissue could lead to an overestimation of some of our lactate concentrations measured by MRS. The variability in this partial volume effect depending on ventricle size and voxel positioning might explain why the agreement between MRS and CSF concentration was so different from case to case (-Fig. 2 ).
Future studies involving tissue segmentation in the attempt to correct for this partial volume effect should be pursued.

Another aspect to be taken into account is that the lactate signal detected by MRS is a doublet (split of the original signal in two peaks separated by $7 \mathrm{~Hz}$ due to Jcoupling). As a doublet, this peak suffers from phase modulation, which gives the LP the singular behavior of inverting at specific TEs, which can aid in its identification but complicates quantification. In our study, we chose the TE of 144 ms suggested in the literature to achieve a more exact phase inversion. ${ }^{19}$ However, other studies have suggested that by measuring the lactate signal at inversion, part of the signal can potentially be lost, and it will be recovered by measuring it at the double TE of $288 \mathrm{~ms}$ (no inversion of the peak). ${ }^{20}$ The recovery of the signal lost by inversion seems to outweigh the signal that would be lost by transverse relaxation by using a longer TE. Therefore, to improve lactate detection in future studies, a TE of $288 \mathrm{~ms}$ should be chosen, and this is even more important at higher fields such as $3 \mathrm{~T}$ to avoid signal loss due to chemical shift artifacts.

\section{Conclusion}

If MRS quantification shows increased lactate levels in the ventricles, CSF puncture is not needed for lactate increase confirmation.

\section{References}

1 Benoist JF, Alberti C, Leclercq S, et al. Cerebrospinal fluid lactate and pyruvate concentrations and their ratio in children: agerelated reference intervals. Clin Chem 2003;49(3):487-494

2 Zhang W-M, Natowicz MR. Cerebrospinal fluid lactate and pyruvate concentrations and their ratio. Clin Biochem 2013;46(7-8):694-697

3 Engelhardt B, Sorokin L. The blood-brain and the blood-cerebrospinal fluid barriers: function and dysfunction. Semin Immunopathol 2009;31(4):497-511

4 Provencher SW. Estimation of metabolite concentrations from localized in vivo proton NMR spectra. Magn Reson Med 1993; 30(6):672-679

5 Inao S, Marmarou A, Clarke GD, Andersen BJ, Fatouros PP, Young HF. Production and clearance of lactate from brain tissue, cerebrospinal fluid, and serum following experimental brain injury. J Neurosurg 1988;69(5):736-744

6 Chi CS, Lee HF, Tsai CR, Chen WS, Tung JN, Hung HC. Lactate peak on brain MRS in children with syndromic mitochondrial diseases. J Chin Med Assoc 2011;74(7):305-309

7 Boddaert N, Romano S, Funalot B, et al. 1H MRS spectroscopy evidence of cerebellar high lactate in mitochondrial respiratory chain deficiency. Mol Genet Metab 2008;93(1):85-88

8 Feng F, You H, Gao J, et al. Evaluation of mitochondrial encephalomyopathy with lactic acidosis and stroke-like episodes with magnetic resonance imaging and proton magnetic resonance spectroscopy. Chin Med Sci J 2006;21(4):234-238

9 Dinopoulos A, Cecil KM, Schapiro MB, et al. Brain MRI and proton MRS findings in infants and children with respiratory chain defects. Neuropediatrics 2005;36(5):290-301

10 Cross JH, Gadian DG, Connelly A, Leonard JV. Proton magnetic resonance spectroscopy studies in lactic acidosis and mitochondrial disorders. J Inherit Metab Dis 1993;16(4):800-811 
11 Lee S-K, Kim J, Kim HD, Lee JS, Lee YM. Initial experiences with proton MR spectroscopy in treatment monitoring of mitochondrial encephalopathy. Yonsei Med J 2010;51(5):672-675

12 Lin DD, Crawford TO, Barker PB. Proton MR spectroscopy in the diagnostic evaluation of suspected mitochondrial disease. AJNR Am J Neuroradiol 2003;24(1):33-41

13 Ibrahim MA, Emerson JF, Cotman CW. Magnetic resonance imaging relaxation times and gadolinium-DTPA relaxivity values in human cerebrospinal fluid. Invest Radiol 1998;33(3): 153-162

14 Sack M, Ende G, Weber-Fahr W. T2-CSF pitfalls using water as an internal reference for metabolite quantification. Proc Intl Soc Magn Reson Med Sci Meet Exhib 2011;2011:19

15 Ernst T, Kreis R, Ross BD. Absolute quantitation of water and metabolites in the human brain. I. Compartments and water. J Magn Reson B 1993;102:1-8
16 Braun KPJ, Gooskens RHJM, Vandertop WP, Tulleken CA, van der Grond J. 1H magnetic resonance spectroscopy in human hydrocephalus. J Magn Reson Imaging 2003;17(3):291-299

17 Isobe T, Matsumura A, Anno I, et al. Lactate quantification by proton magnetic resonance spectroscopy using a clinical MRI machine: a basic study. Australas Radiol 2007;51(4):330-333

18 Bianchi MC, Tosetti M, Battini R, et al. Proton MR spectroscopy of mitochondrial diseases: analysis of brain metabolic abnormalities and their possible diagnostic relevance. AJNR Am J Neuroradiol 2003;24(10):1958-1966

19 Kingsley PB. Scalar coupling and zero-quantum coherence relaxation in STEAM: implications for spectral editing of lactate. Magn Reson Med 1994;31(3):315-319

20 Yablonskiy DA, Neil JJ, Raichle ME, Ackerman JJH. Homonuclear J coupling effects in volume localized NMR spectroscopy: pitfalls and solutions. Magn Reson Med 1998;39(2):169-178 